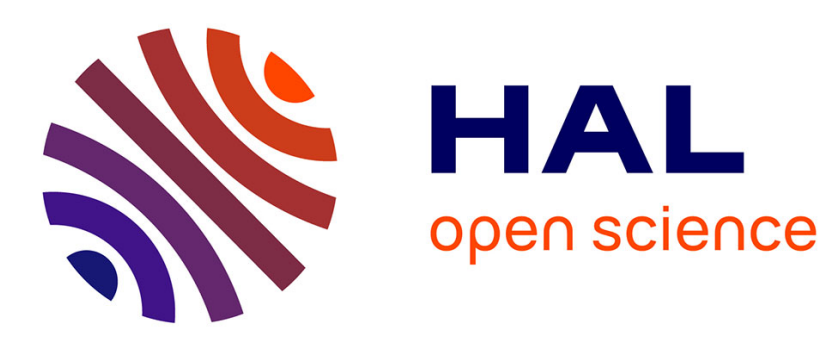

\title{
Stabilization of nonaffine systems: a constructive method for polynomial systems
}

Emmanuel Moulay, W. Perruquetti

\section{To cite this version:}

Emmanuel Moulay, W. Perruquetti. Stabilization of nonaffine systems: a constructive method for polynomial systems. IEEE Transactions on Automatic Control, 2005, 50 (4), pp.520-526. 10.1109/TAC.2005.844899 . hal-03088746

\section{HAL Id: hal-03088746 \\ https://hal.science/hal-03088746}

Submitted on 2 Feb 2021

HAL is a multi-disciplinary open access archive for the deposit and dissemination of scientific research documents, whether they are published or not. The documents may come from teaching and research institutions in France or abroad, or from public or private research centers.
L'archive ouverte pluridisciplinaire HAL, est destinée au dépôt et à la diffusion de documents scientifiques de niveau recherche, publiés ou non, émanant des établissements d'enseignement et de recherche français ou étrangers, des laboratoires publics ou privés. 


\title{
Stabilization of non affine systems: a constructive method for polynomial systems
}

\author{
Emmanuel Moulay and Wilfrid Perruquetti
}

\begin{abstract}
This article focuses on the stabilization of non affine systems described by continuous non linear ordinary differential equations. First, conditions of stabilization using control Lyapunov function give theoretical but non constructive and restrictive results. Secondly, some particular non affine systems are considered: this is polynomial system in the control variable of order two and three (a method is also given for high order systems). The main result is a method of construction of feedbacks for this class of polynomial systems. In the end, the polynomial example of the levitation system is stabilized using an extension of this method to discontinuous feedback.
\end{abstract}

Index Terms-Non affine systems, polynomial systems, stabilization, control Lyapunov function.

\section{INTRODUCTION}

A standard problem in control theory is the stabilization of nonlinear systems. A seminal result is Artstein's theorem [1, Theorem 5.1] which proves, for affine systems, the existence of a control Lyapunov function is equivalent to the existence of a continuous feedback control. This result is a corollary of more general results involving relaxed control (see [1, Theorem 4.1]). For such affine systems, Sontag gives a general formula for the feedback law construction using a control Lyapunov function (see [2]).

Here, the problem is the stabilization of non affine systems. Sufficient conditions of stabilization using control Lyapunov function are addressed in section III. The first result is similar to the one obtained by Artstein in [1, Theorem 4.1] invoking a convexity property which gives a sufficient condition for the existence of an almost continuous stabilizing control, but the proof is non constructive. The second one involving a robustness property is quite restrictive. These methods cannot be generally used for polynomial systems in the control variable. So, we focus in section IV on the class of polynomial systems in the control variable (order two and three). Our main result uses a control Lyapunov function and Cardan formula to construct an explicit stabilizing control. Finally, the section $\mathrm{V}$ contains an example of a polynomial system: the levitation system, for which a discontinuous control is designed by using analogous argument.

This work was supported in part by programs Autoris-TACT 53 and MathsSTIC CNRS 19

E. Moulay is with Laboratoire Paul Painlevé, UFR de Mathématiques Pures et Appliquées, Université des Sciences et Technologies de Lille and LAGIS (UMR-CNRS 8021), Ecole centrale de Lille, Cité Scientifique, 59651 Villeneuve d'Ascq Cedex, France (e-mail: emmanuel.moulay@ec-lille.fr)

W. Perruquetti is with the LAGIS (UMR CNRS 8021), Ecole Centrale de Lille, (e-mail: wilfrid.perruquetti@ec-lille.fr)

\section{Problem FORMulation}

$\epsilon \mathcal{B}^{n}$ is the open ball in $\mathbb{R}^{n}$ centered at the origin of radius $\epsilon>0$ and $u^{[j]}=\left(u_{1}^{\alpha_{1}}, \ldots, u_{m}^{\alpha_{m}}\right)$ where $j$ and $\alpha_{1}, \ldots, \alpha_{m}$ are integers such that $\alpha_{1}+\ldots+\alpha_{m}=j$. If $E$ is a non empty subset of $\mathbb{R}^{n}, c l(E)$ denotes its closure and $\overline{c o}(E)$ the smallest closed convex set containing $E . \mathcal{U}$ denotes a non empty open set of $\mathbb{R}^{m}$ containing the origin and $\mathcal{V}$ a neighborhood of the origin in $\mathbb{R}^{n}$. Let $\mathcal{C P}$ be the set of positive definite continuous functions $V: \mathbb{R}^{n} \rightarrow \mathbb{R}_{\geq 0}$ with continuous partial derivatives. Let $f: \mathbb{R}^{n} \times \mathcal{U} \rightarrow \mathbb{R}^{n}$ be a continuous function such that $f(0,0)=0$ with which one associates the following system

$$
\dot{x}=f(x, u), \quad x \in \mathbb{R}^{n} \text { and } u \in \mathcal{U} .
$$

Under smoothness hypothesis of $f$, a polynomial expansion of this function with respect to the control variable will be

$$
\dot{x}=f_{0}(x)+\sum_{j=1}^{k} f_{j}(x) u^{[j]}+R(x, u),
$$

with $x \in \mathbb{R}^{n}$ and $u \in \mathcal{U}^{\prime} \subset \mathcal{U}$. The general stabilization problem of such a system is a challenge, which becomes tractable in the case $k \leq 3$ and $R(x, u)=0$ (that will be considered later on (see section IV)).

Definition 1: System (1) is almost stabilizable, if there exists a feedback control law $u: \mathcal{V} \rightarrow \mathcal{U}$ continuous on $\mathcal{V} \backslash\{0\}$ such that:

- $u(0)=0$,

- the origin is a uniformly asymptotically stable ${ }^{1}$ equilibrium of the closed-loop system:

$$
\dot{x}=f(x, u(x)), \quad x \in \mathcal{V} .
$$

Moreover, if $u$ is continuous on $\mathcal{V}$, we say that the system (1) is stabilizable.

Definition 2: The system (1) is globally stabilizable if $\mathcal{V}=$ $\mathbb{R}^{n}$ in Definition 1.

Let $V \in \mathcal{C P}, V$ is said to be a control Lyapunov function of (1) if:

- $V(0)=0$

- $\forall x \in \mathcal{V} \backslash\{0\}, \inf _{u \in \mathbb{R}^{m}}\langle\nabla V(x), f(x, u)\rangle<0$.

The lie derivative of $V: \mathbb{R}^{n} \rightarrow \mathbb{R}$ along $f: \mathbb{R}^{n} \rightarrow \mathbb{R}^{n}$ is defined by:

$$
\mathcal{L}_{f} V: \mathbb{R}^{n} \rightarrow \mathbb{R}, \quad \mathcal{L}_{f} V(x)=\langle\nabla V(x), f(x)\rangle .
$$

A control Lyapunov function for the system (1) $V: \mathcal{V} \rightarrow \mathbb{R}_{\geq 0}$ satisfies the small control property if for each $\epsilon>0$ there

\footnotetext{
${ }^{1}$ The origin of the system (3) is uniformly asymptotically stable if it is uniformly stable and uniformly attractive.
} 
is $\delta>0$ such that, if $x \in \delta \mathcal{B}^{n} \backslash\{0\}$, then there is at least $u \in \epsilon \mathcal{B}^{m}$ such that $\langle\nabla V(x), f(x, u)\rangle<0$.

\section{StABILIZATION USING CONTROL LyAPUNOV FUNCTION}

The stabilization problem of affine systems involving the control Lyapunov functions is described by Artstein in [1, Theorem 5.1]. He also gives a necessary and sufficient condition for the stabilization of non affine systems involving relaxed controls which are probability measures (see [1, Theorem 4.1]). Here, using a convexity assumption, a sufficient condition is obtained to guarantee the existence of a continuous stabilizing control (except possibly at the origin) for non affine systems. For this, one needs the convexity ${ }^{2}$ of $u \mapsto\langle\nabla V(x), f(x, u)\rangle$ for all $x \in \mathcal{V} \backslash\{0\}$, where $V$ is a control Lyapunov function.

Proposition 3: If there exists a control Lyapunov function $V$ for the system (1) such that $u \mapsto\langle\nabla V(x), f(x, u)\rangle$ is convex for all $x \in \mathcal{V} \backslash\{0\}$, then the system (1) is almost stabilizable. In addition, if $V$ satisfies the small control property then the system (1) is stabilizable.

Proof. There exists a neighborhood $\mathcal{V}$ of the origin such that for all $x \in \mathcal{V} \backslash\{0\}$, one defines the set valued function ${ }^{3}$ $\Phi: \mathcal{V} \backslash\{0\} \rightarrow 2^{\mathcal{U}}, x \mapsto \Phi(x)$ where $2^{\mathcal{U}}$ will denote the family of non-empty subsets of $\mathcal{U}$ and:

$$
\Phi(x)=\{v \in \mathcal{U}:\langle\nabla V(x), f(x, v)\rangle<0\} .
$$

As $\Phi(x)$ is a non-empty, closed, convex set for all $x \in \mathcal{V} \backslash\{0\}$, and $\Phi$ is lower semi- continuous ${ }^{4}$, one may apply Michael's theorem (see [3] or [4, Chapter 1, section 11]) to find a continuous selection $u: \mathcal{V} \backslash\{0\} \rightarrow \mathcal{U}$ such that $u(0)=0$. Then, $V$ is a Lyapunov function for the closed loop system (3).

If $V$ satisfies the small control property, one may assume that $\lim _{x \rightarrow 0} u(x)=0$ as it is shown in [1].

If the system is affine in the control, a control Lyapunov function is always a convex control Lyapunov function. Moreover, one may notice that if $\langle\nabla V(x), f(x, u)\rangle$ is a quadratic form $\langle\nabla V(x), f(x, u)\rangle=u^{T} Q(x) u$ with $Q(x)$ a positive definite function, then $u \mapsto\langle\nabla V(x), f(x, u)\rangle$ is convex. It is important to note that when dealing with (2) the set is not convex in general. This is an obstacle for the generalization of Sontag's formula.

One gives an example of a non affine system which is known to be almost stabilizable with the proposition 3 .

Example 4: Let us consider the system

$$
\left\{\begin{array}{l}
\dot{x}_{1}=-x_{1}-x_{2} \\
\dot{x}_{2}=x_{1}+x_{2} g(u)
\end{array}\right.
$$

${ }^{2} \mathrm{~A}$ function $f: \mathbb{R}^{n} \rightarrow \mathbb{R}^{n}$ is convex if for all $(x, y) \in \mathbb{R}^{n} \times \mathbb{R}^{n}$ and $\lambda \in[0,1]$,

$$
f(\lambda x+(1-\lambda) y) \leq \lambda f(x)+(1-\lambda) f(y) .
$$

\footnotetext{
${ }^{3} \mathrm{~A}$ set valued function $\Phi$ on the vector space $\mathcal{E}$ to the vector space $\mathcal{F}$ is a function that associates with any $x \in \mathcal{E}$ a subset $\Phi(x)$ of $\mathcal{F}$.

${ }^{4} \Phi$ is lower semi-continuous if $\{x \in \mathcal{E}: \Phi(x) \cap O \neq \emptyset\}$ is open in $\mathcal{E}$ for every open $O \subset \mathcal{F}$.
}

where $u \in \mathbb{R}, g$ is a convex function such that $g(0)=$ 0 , and the function $V(x)=\frac{1}{2}\left(x_{1}^{2}+x_{2}^{2}\right)$. One sees that $\langle\nabla V(x), f(x, u)\rangle=-x_{1}^{2}+x_{2}^{2} g(u)$. For $x \neq 0$, $\inf _{u \in \mathbb{R}}\langle\nabla V(x), f(x, u)\rangle<0$, so $V$ is a control Lyapunov function for the system. For all $x \in \mathbb{R}^{2} \backslash(0,0), u \mapsto-x_{1}^{2}+x_{2}^{2} g(u)$ is convex, so using the proposition 3 , one knows that the system is almost stabilizable. For example, if $g(u)=u^{2}-2 u$, the system is stabilizable by the continuous feedback $u(x)=$ $\frac{2 x_{2}^{2}}{1+x_{2}^{2}}$.

The stabilization of affine systems is well known (see [1], [2]). Now, we want to use a control Lyapunov function with a robustness property to stabilize non affine systems. Let us consider the system

$$
\dot{x}=f_{0}(x)+f_{1}(x) u+R(x, u),
$$

where $x \in \mathbb{R}^{n}, u \in \mathcal{U}, f_{1}=\left(f_{1, j}\right)_{1 \leq j \leq m}$ and $R$ are continuous. One supposes that there exists $u_{0}=\left(u_{0,1}, \ldots, u_{0, m}\right) \in \mathcal{U}$ such that

$$
f_{0}(0)+\sum_{j=1}^{m} f_{1, j}(0) u_{0, j}+R\left(0, u_{0}\right)=0 .
$$

With the system (4), we associate the affine system

$$
\dot{x}=f_{0}(x)+\sum_{i=1}^{m} f_{1, i}(x) u_{i} .
$$

and defines

$$
\begin{aligned}
a(x) & =\mathcal{L}_{f_{0}} V(x) \\
b_{i}(x) & =\mathcal{L}_{f_{i}} V(x), \quad 1 \leq i \leq m .
\end{aligned}
$$

Now, one can give a sufficient condition for stabilization of the system (4).

Proposition 5: If there exists a control Lyapunov function $V$ for the affine system (5) such that for all $x \in \mathcal{V}$, and all $u \in \mathcal{U}$,

$$
\langle\nabla V(x), R(x, u)\rangle \leq 0
$$

then the two systems (5) and (4) are almost stabilizable by the same feedback control law. In addition, if the control Lyapunov function satisfies the small control property then the system (4) is stabilizable.

Proof. There exists a neighborhood $\mathcal{V}$ of the origin such that for all $x \in \mathcal{V} \backslash\{0\}$, one defines the set valued function $\Psi: \mathcal{V} \backslash\{0\} \rightarrow 2^{\mathcal{U}}, x \mapsto \Phi(x)$ where $2^{\mathcal{U}}$ will denote the family of non-empty subsets of $\mathcal{U}$ and:

$$
\Psi(x)=\left\{v \in \mathcal{U}: a(x)+\sum_{i=1}^{m} b_{i}(x) v_{i}<0\right\} .
$$

As $\Psi(x)$ is a non-empty, closed, convex set for all $x \in \mathcal{V} \backslash\{0\}$, and $\Psi$ is lower semi- continuous, one may apply Michael's theorem [3], [4] to extract a continuous selection $u: \mathcal{V} \backslash\{0\} \rightarrow$ $\mathcal{U}$ (that is a continuous function $u$ on $\mathcal{V} \backslash\{0\}$ such that $u(x) \in$ $\Psi(x)$ ), extended by $u(0)=0$. So, $V$ is a Lyapunov function for the closed loop system associated with the system (5). Thus, for all $x \in \mathcal{V} \backslash\{0\}$,

$$
a(x)+\sum_{i=1}^{m} b_{i}(x) u_{i}(x)+\langle\nabla V(x), R(x, u(x))\rangle<0 .
$$


So, $V$ is a Lyapunov function for the closed-loop system (4), and using the Lyapunov theorem one knows that the origin of the closed loop system associated with the system (4) is asymptotically stable.

Example 6: Let us consider the system

$$
\left\{\begin{array}{l}
\dot{x}_{1}=x_{1} x_{2}-x_{1} h_{1}(u) \\
\dot{x}_{2}=-x_{2}+u-x_{2} h_{2}(u)
\end{array}\right.
$$

where $h_{i}: \mathbb{R} \rightarrow \mathbb{R}_{\geq 0}$ are positive definite. One sees that the smooth function

$$
V(x)=\frac{x_{1}^{2} e^{2 x_{2}}+x_{2}^{2}}{2} .
$$

is a control Lyapunov function for the affine system

$$
\left\{\begin{array}{l}
\dot{x}_{1}=x_{1} x_{2} \\
\dot{x}_{2}=-x_{2}+u
\end{array}\right.
$$

Let $R(x, u)=\left(-x_{1} h_{1}(u),-x_{2} h_{2}(u)\right)$, for all $x \in \mathbb{R}^{2}$ and all $u \in \mathbb{R}$, we have

$\langle\nabla V(x), R(x, u)\rangle=-x_{1}^{2} e^{2 x_{2}} h_{1}(u)-\left(x_{2}^{2} x_{1}^{2}+x_{2}^{2}\right) h_{2}(u) \leq 0$.

Thus, using the proposition 5, one knows that the system 6 is almost stabilizable by the Sontag's feedback control given in [2]

$$
u(x)=\frac{-x_{2}^{2}+\sqrt{x_{2}^{4}+\left(x_{1}^{2} e^{2 x_{2}}+x_{2}\right)^{4}}}{x_{1}^{2} e^{2 x_{2}}+x_{2}} .
$$

which stabilizes the affine system 7 .

The following exemple emphasizes that the proposition (5) is not generally well adapted for polynomial systems in the control variable.

Example 7: Let us consider the system

$$
\dot{x}=x e^{2 x}+\left(2 x^{2}+e^{2 x}\right) u+x u^{2} .
$$

All smooth positive definite scalar functions are equivalent to the function $V(x)=\frac{x^{2}}{2}$. Then $\langle\nabla V(x), R(x, u)\rangle=x^{2} u^{2} \geq$ 0 is not negative. Thus, the proposition 5 fails to stabilize the system for all control Lyapunov functions. Nevertheless, such a system is stabilized in example 9 with our novel control design tool for polynomial systems in the control variable developed in the following section IV.

\section{Polynomial systems}

Nowadays, the class of polynomial systems appears in many practical fields, as in magnetic problems (see section V). There exists two main difficulties in the use of the two propositions 3 and 5. The first one is that the feedback control is not constructive in the proposition 3. The second one is that the class of systems which are known to be stabilizable by propositions 3 and 5 does not contain the important class of polynomial systems. Indeed, the convexity and robustness properties exclude a large part of the class of polynomial systems, as it can be seen in example 4, 6 and 7. So, we want to give results for constructing controllers for polynomial systems, mainly for order two and three cases. We use algebra formulae to find a feedback control by radical, when it is possible.

\section{A. Order two control systems}

Let us consider the polynomial system

$$
\dot{x}=f_{0}(x)+f_{1}(x) u+f_{2}(x) u^{2},
$$

where $x \in \mathbb{R}^{n}, u \in \mathbb{R}, f_{i}: \mathbb{R}^{n} \rightarrow \mathbb{R}^{n}$ and $f_{0}(0)=0$. For a positive definite function $V$, let us introduce:

$$
\begin{aligned}
& a(x)=\mathcal{L}_{f_{2}} V(x), \\
& b(x)=\mathcal{L}_{f_{1}} V(x), \\
& c(x)=\mathcal{L}_{f_{0}} V(x) .
\end{aligned}
$$

First of all, let us note that if there exists a positive definite function $V$ such that for all $x \in \mathcal{V}$,

$$
b(x)^{2}-4 a(x) c(x)<0 \text { and } a(x)<0
$$

then the system (8) is stabilizable by any feedback. Indeed, we know that for all $x \in \mathcal{V}$ and all $u \in \mathbb{R}$,

$$
a(x) u^{2}+b(x) u+c(x)<0 .
$$

So, we may choose every control, in particular no control

$$
u(x)=0, \quad x \in \mathcal{V} .
$$

Proposition 8: If there exists $V \in \mathcal{C P}$ such that for all $x \in \mathcal{V} \backslash\{0\}, b(x)^{2}-4 a(x) c(x)>0, a(x) \neq 0$, then the system (8) is almost stabilizable with control

$$
u(x)=\left\{\begin{array}{lll}
w(x) & \text { if } & x \in \mathcal{V} \backslash\{0\} \\
0 & \text { if } & x=0
\end{array}\right.
$$

where $w(x)=\frac{-b(x)+\sqrt{b(x)^{2}-4 a(x)(c(x)+\varphi(x))}}{2 a(x)}$ and $\varphi$ is a continuous positive definite function such that for all $x \in \mathcal{V} \backslash\{0\}, b(x)^{2}-4 a(x) c(x) \geq 4 a(x) \varphi(x)$.

Proof. Let $\varphi$ be a continuous function on $x \in \mathcal{V} \backslash\{0\}$, positive definite such that for all $x \in \mathcal{V} \backslash\{0\}$,

$$
b(x)^{2}-4 a(x) c(x) \geq 4 a(x) \varphi(x) .
$$

Since $b(x)^{2}-4 a(x) c(x)>0$, one may choose $\varphi$ defined by

$$
\varphi(x)=\left\{\begin{array}{ll}
\frac{b(x)^{2}-4 a(x) c(x)}{4|a(x)|} & \text { if } \quad x \in \mathcal{V} \backslash\{0\} . \\
0 & \text { if } \quad x=0
\end{array} .\right.
$$

Then, the control defined by (9) satisfies the condition: $\left.\dot{V}(x)\right|_{(8),(9)}=a(x) u(x)^{2}+b(x) u(x)+c(x)=-\varphi(x)$ for all $x \in \mathcal{V} \backslash\{0\}$. Thus, $u(x)$ is an almost smooth feedback control for the system.

Example 9: Let us consider the system

$$
\dot{x}=x e^{2 x}+\left(2 x^{2}+e^{2 x}\right) u+x u^{2}
$$

and the function $V(x)=\frac{x^{2}}{2}$. We have for all $x \in \mathbb{R}^{2} \backslash\{0\}$,

$$
\begin{aligned}
a(x) & =x^{2} \neq 0, \\
b(x)^{2}-4 a(x) c(x) & =4 x^{6}+x^{2} e^{4 x}>0 .
\end{aligned}
$$

If we set the positive definite function

$$
\psi(x)=\left\{\begin{array}{ccc}
\frac{1}{4}\left(e^{4 x}-1\right) & \text { if } & x \geq 0 \\
-\frac{1}{4 e^{4}} x & \text { if } & -1 \leq x \leq 0 \\
\frac{1}{4} e^{4 x} & \text { if } & x \leq-1
\end{array}\right.
$$


then $\psi(x) \leq \frac{e^{4 x}}{4} \cdot \varphi(x)=x^{4}+\psi(x)$ is a positive definite function such that $b(x)^{2}-4 a(x) c(x) \geq 4 a(x) \varphi(x)$, i.e. such that $\varphi(x) \leq x^{4}+\frac{e^{4 x}}{4}$. So one may apply the proposition 8 and thus

$$
u(x)=\frac{-2 x^{3}-x e^{2 x}+\sqrt{4 x^{6}+x^{2}\left(e^{4 x}-4 \varphi(x)\right)}}{2 x^{2}}
$$

is a feedback control for the system and leads to the following simulation given in figure 1 .

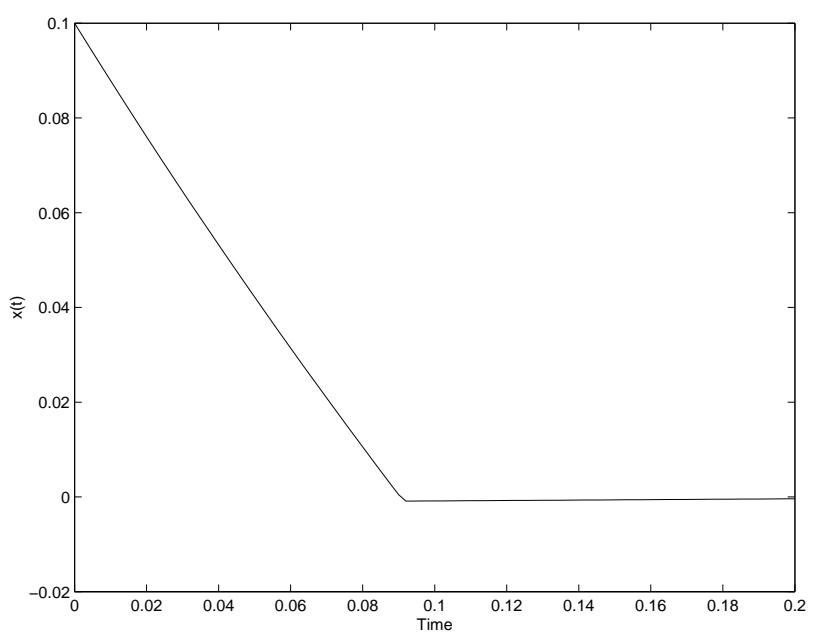

Fig. 1. Simulation of $x(t)$ under the feedback control $u(t)$

There exists another case, when it is possible to build a continuous feedback:

Proposition 10: If there exists a control Lyapunov function $V$ for the system (8) such that for all $x \in \mathcal{V} \backslash\{0\}$,

$$
b(x)^{2}-4 a(x) c(x) \geq 4 a(x) \sqrt{a(x)^{2}+b(x)^{4}+c(x)^{2}}
$$

then the system (8) is almost stabilizable with control

$$
\begin{gathered}
u(x)= \begin{cases}-\varphi_{1}\left(a(x), b(x)^{2}, c(x)\right) & \text { if } \quad x \neq 0 \\
0 & \text { if } \quad x=0\end{cases} \\
\varphi_{1}(a, b, c)=\left\{\begin{array}{lll}
g(a, b, c) & \text { if } \quad a \neq 0 \\
\frac{c+\sqrt{b^{2}+c^{2}}}{b} & \text { if } \quad a=0 \text { and } b \neq 0 \\
0 & \text { if } \quad a=b=0
\end{array}\right.
\end{gathered}
$$

where $g(a, b, c)=\frac{b-\sqrt{b^{2}-4 a\left(c+\sqrt{a^{2}+b^{2}+c^{2}}\right)}}{2 a}$.

Proof. Let $(a, b, c) \in S$ with $S=S_{1} \cup S_{2}$ with $S_{1}=\left\{(a, b, c) \in \mathbb{R}^{3}: a \neq 0\right\} \quad$ and $\quad S_{2}=$ $\left\{(0, b, c) \in \mathbb{R}^{3}: b>0\right.$ or $\left.c<0\right\}$. Then $\varphi_{1}$ is continuous on $S$. By assumption, we know that $(a(x), b(x), c(x)) \in S$. Thus, control (11) is a continuous feedback control for the system (8) Indeed,

$$
a(x) u(x)^{2}+b(x) u(x)+c(x)=-\sqrt{a(x)^{2}+b(x)^{4}+c(x)^{2}}
$$

which is negative definite.

The multi-input case is much more difficult but for the following special case it is very close to the single-input case. One considers the system:

$$
\dot{x}=f_{0}(x)+\sum_{j=1}^{m} f_{1, j}(x) u_{j}+\sum_{j=1}^{m} f_{2, j}(x) u_{j}^{2},
$$

where $x \in \mathbb{R}^{n}, u \in \mathbb{R}^{m}, f_{i, j}: \mathbb{R}^{n} \rightarrow \mathbb{R}^{n}$ and $f_{0}(0)=0$. For a positive definite function $V$, one defines

$$
\begin{aligned}
a_{j}(x) & =\mathcal{L}_{f_{2, j}} V(x), \\
b_{j}(x) & =\mathcal{L}_{f_{1, j}} V(x), \\
c(x) & =\mathcal{L}_{f_{0}} V(x) .
\end{aligned}
$$

Proposition 11: If there exists $V \in \mathcal{C P}$ such that for all $x \in \mathcal{V} \backslash\{0\}$ and all $j \in\{1, \ldots, m\}$,

$$
\begin{aligned}
& b_{j}(x)^{2}-4 a_{j}(x) c(x)>0, \\
& a_{j}(x)=\mathcal{L}_{f_{2, j}} V(x) \neq 0,
\end{aligned}
$$

then the system (12) is almost stabilizable (see the following proof for the construction of the controls).

Proof. We can build a continuous function $\varphi$ on $x \in \mathcal{V} \backslash\{0\}$, positive definite such that for all $x \in \mathcal{V} \backslash\{0\}$ and all $j \in$ $\{1, \ldots, m\}$,

$$
b_{j}(x)^{2}-4 a_{j}(x) c(x) \geq 4 a_{j}(x) \varphi(x)
$$

For example, one may take:

$$
\varphi(x)=\min _{1 \leq j \leq m} \frac{b_{j}(x)^{2}-4 a_{j}(x) c(x)}{4\left|a_{j}(x)\right|} .
$$

The control:

$$
u_{j}(x)=\left\{\begin{array}{lll}
w_{j}(x) & \text { if } & x \in \mathcal{V} \backslash\{0\} \\
0 & \text { if } & x=0
\end{array}\right.
$$

with $w_{j}(x)=\frac{-b_{j}(x)+\sqrt{b_{j}(x)^{2}-4 a_{j}(x)(c(x)+\varphi(x))}}{2 a_{j}(x)}$ satisfies for all $x \in \mathcal{V} \backslash\{0\}$ :

$$
c(x)+\sum_{j=1}^{m} b_{j}(x) u_{j}(x)+\sum_{j=1}^{m} a_{j}(x) u_{j}^{2}(x)=-m \varphi(x) .
$$

So, $u(x)$ is a feedback control for the system (12).

\section{B. Order three control systems}

Here, we consider the polynomial system

$$
\dot{x}=f_{0}(x)+f_{1}(x) u+f_{2}(x) u^{2}+f_{3}(x) u^{3},
$$

where $x \in \mathbb{R}^{n}, u \in \mathbb{R}, f_{i}: \mathbb{R}^{n} \rightarrow \mathbb{R}^{n}$ are continuous and $f_{0}(0)=0$. For a positive definite function $V$, one defines

$$
\begin{aligned}
a(x) & =\mathcal{L}_{f_{3}} V(x) \\
b(x) & =\mathcal{L}_{f_{2}} V(x) \\
c(x) & =\mathcal{L}_{f_{1}} V(x) \\
d(x) & =\mathcal{L}_{f_{0}} V(x) \\
p(x) & =\frac{3 a(x) c(x)-b(x)^{2}}{3 a(x)^{2}} \\
q(x) & =\frac{d(x)}{a(x)}-\frac{b(x) c(x)}{3 a(x)^{2}}+\frac{2}{27} \frac{b(x)^{3}}{a(x)^{3}} \\
\Delta(x) & =4 p(x)^{3}+27 q(x)^{2} .
\end{aligned}
$$

Proposition 12: If there exists $V \in \mathcal{C P}$ such that for all $x \in \mathcal{V} \backslash\{0\}, \Delta(x)>0$ and $a(x) \neq 0$, then the system (13) is almost stabilizable. 
Proof. We know that there exists a continuous function $\varphi$ on $x \in \mathcal{V} \backslash\{0\}$, positive definite such that for all $x \in \mathcal{V} \backslash\{0\}$,

$$
c(x)^{2}-4 b(x) d(x) \geq 4 b(x) \varphi(x) .
$$

Let

$$
\tilde{q}(x)=\frac{d(x)+\varphi(x)}{a(x)}-\frac{b(x) c(x)}{3 a(x)^{2}}+\frac{2}{27} \frac{b(x)^{3}}{a(x)^{3}}
$$

and we have for all $x \in \mathcal{V} \backslash\{0\}$ :

$$
\tilde{\Delta}(x)=4 p(x)^{3}+27 \tilde{q}(x)^{2} \geq \Delta(x) \geq 0 .
$$

Using the Cardan formula, we know that:

$$
u(x)=\left\{\begin{array}{lll}
v(x) & \text { if } & x \in \mathcal{V} \backslash\{0\} \\
0 & \text { if } \quad x=0
\end{array}\right.
$$

with $v(x)=\sqrt[3]{-\frac{\tilde{q}(x)}{2}+\sqrt{\frac{\tilde{q}(x)^{2}}{4}+\frac{p(x)^{3}}{27}}}+$ $\sqrt[3]{-\frac{\tilde{q}(x)}{2}-\sqrt{\frac{\tilde{q}(x)^{2}}{4}+\frac{p(x)^{3}}{27}}}$ is an almost smooth feedback control.

If $c(x)^{2}-4 b(x) d(x)$ is positive definite, one may choose $\varphi$ as follows:

$$
\varphi(x)=\left\{\begin{array}{ll}
\frac{c(x)^{2}-4 b(x) d(x)}{4|b(x)|} & \text { if } \quad x \in \mathcal{V} \backslash\{0\} . \\
0 & \text { if } \quad x=0
\end{array} .\right.
$$

Let us give an academic example using the previous corollary.

Example 13: We consider the system

$$
\dot{x}=x-2 u^{3} \quad x, u \in \mathbb{R} .
$$

Using $V(x)=\frac{x^{2}}{2}$ and $\varphi(x)=x^{2}$, we have for all $x \in \mathbb{R} \backslash\{0\}$, $a(x)=-2 x \neq 0$. We know with the proposition (12) that $u(x)=\sqrt[3]{x}$ is a feedback control for the system. Moreover, here $u$ is continuous at the origin.

\section{High order systems}

Now, we consider the system

$$
\dot{x}=f_{0}(x)+f_{1}(x) u+\ldots+f_{p}(x) u^{p},
$$

where $x \in \mathbb{R}^{n}, u \in \mathbb{R}, f_{i}: \mathbb{R}^{n} \rightarrow \mathbb{R}^{n}$ are continuous and $f_{0}(0)=0$. For a positive definite function $V$, one defines $a_{i}(x)=\mathcal{L}_{f_{i}} V(x)$. The problem is to find $V$ a positive definite continuous function and $\varphi$ a positive definite continuous function outside the origin such that for all $x \neq 0$,

$$
\varphi(x)+a_{0}(x)+a_{1}(x) u+\ldots+a_{p}(x) u^{p}=0 .
$$

For such general system (15) with $p \geq 4$, we have no hope to have a formula by radical for a feedback control. But since the polynomial $P(x)[u]=\varphi(x)+a_{0}(x)+a_{1}(x) u+\ldots+$ $a_{p}(x) u^{p}$ belongs to the ring $C^{0}(\mathbb{R})[u]$, it is sometimes possible to split $P(x)[u]$ into several polynomials in $C^{0}(\mathbb{R})[u]$ with small degree. The main goal is to split $P(x)[u]$ in the following sense

$$
P(x)[u]=P_{1}(x)[u] P_{2}(x)[u]
$$

where $1 \leq \operatorname{deg}_{u} P_{1}(x)[u] \leq 3$ and to apply, if it is possible, the previous results to find $u(x)$ such that $P_{1}(x)[u(x)]=0$. Thus, $u(x)$ will be a feedback for the general system (15).

If $a_{i}$ are more regular and if $\varphi$ may be chosen more regular (in particular analytic), one may apply general theorems on polynomial decomposition (a survey is presented in [5]).

\section{AN EXTENSION tO DisCONTINUOUS CONTROLLER}

In this section, we will give a method to extend proposition 8 to discontinuous controllers. The same is true for proposition 12. Let us consider the system (8) and the sets $\mathcal{E}=$ $\{x \in \mathcal{V}: a(x) \neq 0\}, \mathcal{F}=\{x \in \mathcal{V}: a(x)=0$ and $b(x) \neq 0\}$ and $\mathcal{G}=\{x \in \mathcal{V}: a(x)=0$ and $b(x)=0\}$. If there exists $V \in \mathcal{C P}$ such that for all $x \in \mathcal{V} \backslash\{0\}, b(x)^{2}-4 a(x) c(x) \geq 0$, then we set

$$
u(x)=\left\{\begin{array}{lll}
w(x) & \text { if } \quad x \in \mathcal{E} \backslash\{0\} \\
\frac{-c(x)-\psi(x)}{b(x)} & \text { if } \quad x \in \mathcal{F} \backslash\{0\} \\
0 & \text { if } \quad x \in \mathcal{G} \cup\{0\}
\end{array}\right.
$$

where $w(x)$ is given by (9) with $\varphi$ a continuous positive function on $\mathcal{E} \backslash\{0\}$ such that for all $x \in \mathcal{E} \backslash\{0\}$,

$$
b(x)^{2}-4 a(x) c(x) \geq 4 a(x) \varphi(x)
$$

and $\psi$ is a continuous positive function on $\mathcal{F} \backslash\{0\}$. If $u$ is continuous, one tries to conclude with the theorem of LaSalle for differential equations (see [6, Theorem 4.4]). If $u$ is discontinuous, one considers the differential inclusion

$$
\dot{x} \in \overline{c o}\left(\bigcap_{\epsilon>0} f(x, u(x+\epsilon \overline{\mathcal{B}}))\right)
$$

which solutions are called Krasovskii solutions (for more details on differential inclusions see [7]). Then one uses, when it is possible, the LaSalle's theorem for differential inclusions in [8, Theorem 3.2] to conclude that $u$ stabilizes the system (8). Let us give the example of the magnetic levitation system.

Example 14: We consider a metallic ball within a magnetic field derived from a coil. The current feeding the coil is the control variable and the goal is to maintain the ball in levitation at a desired position with respect to the ground. The most famous application is high-speed ground transportation systems. The levitation system is described by the following equation (see [9] for more details)

$$
\left\{\begin{array}{l}
\dot{e}_{1}=e_{2} \\
\dot{e}_{2}=g-\frac{k_{0}}{\left(l_{0}+e_{1}+x_{1 \text { ref }}\right)^{2}} u^{2}
\end{array}\right.
$$

where $e_{1}$ is the error between $x_{1}$ the vertical position of the ball with respect to the ground and $x_{1 \text { ref }}$ (a constant desired vertical position), $e_{2}=\dot{e}_{1}=\dot{x}_{1}$ is the speed, $g$ is the gravity acceleration and $k_{0}$ and $l_{0}$ are some positive parameters. Let us consider the $\mathcal{C P}$ function $V(x)=\alpha x_{1}^{2}+x_{2}^{2}$ with $0<$ $\alpha<20$ and let us restrict our attention to the following set $\mathcal{U}=\left\{x \in \mathbb{R}^{2}: V(x) \leq \frac{g^{2}}{4 \alpha^{2}}\right\}$ which is reasonable knowing that in practice $\left|e_{1}\right|<\frac{g}{20}$. One sees that

$$
b(e)^{2}-4 a(e) c(e)=\frac{16 e_{2}^{2} k_{0}}{\left(l_{0}+e_{1}+x_{1 r e f}\right)^{2}}\left(\alpha e_{1}+g\right) .
$$


Since we restrict our attention to $\mathcal{U}$, one gets $\left|e_{1}\right| \leq \frac{g}{2 \alpha}$. One deduces that, within the set $\mathcal{U}$ we obtain $b(e)^{2}-4 a(e) c(e)>0$ for $e_{2} \neq 0$. With the proposition (8), one knows that

$$
v_{\varphi}(e)=-\frac{\left|l_{0}+e_{1}+x_{1 r e f}\right|}{e_{2}} \sqrt{\frac{e_{2}\left(e_{2}\left(e_{1}+g\right)+\varphi(e)\right)}{k_{0}}}
$$

is a continuous feedback for the system outside the manifold

$$
\mathcal{M}=\left\{x \in \mathbb{R}^{2}: x_{2}=0\right\}
$$

where $\varphi$ is a continuous positive function outside $\mathcal{M}$ such that

$$
e_{2} \varphi(e) \geq-4 e_{2}^{2}\left(e_{1}+g\right) \text {. }
$$

If one chooses

$$
\varphi(e)=\beta\left|e_{2}\right|\left(\alpha e_{1}+g\right),
$$

with $\beta$ such that $\left(\alpha e_{1}+g\right)\left(1+\beta \operatorname{sgn}\left(e_{2}\right)\right)>0$ (in practice $0<\beta<1)$ then one sees that $u(e)=v_{\beta\left|e_{2}\right|\left(\alpha e_{1}+g\right)}(e)=$ $-\left|l_{0}+e_{1}+x_{1 r e f}\right| \operatorname{sgn}\left(e_{2}\right) \sqrt{\frac{\left(\alpha e_{1}+g\right)\left(1+\beta \operatorname{sgn}\left(e_{2}\right)\right)}{k_{0}}} \quad$ stabilizes the levitation system outside $\mathcal{M}$. The problem is that $u$ is not continuous everywhere. Let

$$
\mathcal{S G N}(x)=\left\{\begin{array}{ccc}
-1 & \text { if } & x<0 \\
{[-1,1]} & \text { if } & x=0 \\
1 & \text { if } & x>0
\end{array}\right.
$$

we have for all $v \in\left(e_{2}, g-\left(\alpha e_{1}+g\right)\left(1+\beta \mathcal{S G N}\left(e_{2}\right)\right)\right)$, $\langle\nabla V(e), v\rangle=-\beta\left|e_{2}\right|\left(\alpha e_{1}+g\right)$. Applying the Lyapunov theorem for differential inclusions (see [10, Theorem 8.2] or [7]), one deduces that the closed loop system associated with the levitation system

$$
\left\{\begin{array}{l}
\dot{e}_{1}=e_{2} \\
\dot{e}_{2} \in g-\left(\alpha e_{1}+g\right)\left(1+\beta \mathcal{S G N}\left(e_{2}\right)\right)
\end{array}\right.
$$

is stable everywhere and asymptotically stable outside the variety $\mathcal{M}$. Let us consider the set $\mathcal{S}=\operatorname{cl}\left\{e \in \mathbb{R}^{2}:\langle\nabla V(e), v\rangle=\right.$ 0 for all $\left.v \in\left(e_{2}, g-\left(\alpha e_{1}+g\right)\left(1+\beta \mathcal{S G N}\left(e_{2}\right)\right)\right)\right\}$, then $\mathcal{S}=\mathbb{R} \times\{0\}$ and the largest invariant subset of $\mathcal{S}$ is $(0,0)$. Using LaSalle's theorem for differential inclusions (see [8]), one deduces that the closed-loop levitation system is asymptotically stable. Thus $u(e)$ stabilizes the levitation system and leads to the following simulation for $(\alpha, \beta)=(15,0.01)$ on figure 2 .

Remark 15: $e_{1}$ reaches the origin in $7 s$ with a degree of precision of the order of $\pm 3 \cdot 10^{-3} \mathrm{~m}$. The time of convergence and the degree of precision can be adjusted by the positive constants $(\alpha, \beta)$.

\section{CONCLUSION}

This article presents different ways to study the problem of stabilization of continuous non affine systems. If the problem of stabilization of affine systems is now well known and exhibits a universal formula (due to Sontag), the problem of stabilization of more general non affine systems has not yet a universal construction and is an active research field. For such systems, we provide that continuous feedback stabilizer for system polynomial in the control variable up to order three. And derived from these results, discontinuous feedback controllers are also underlined.
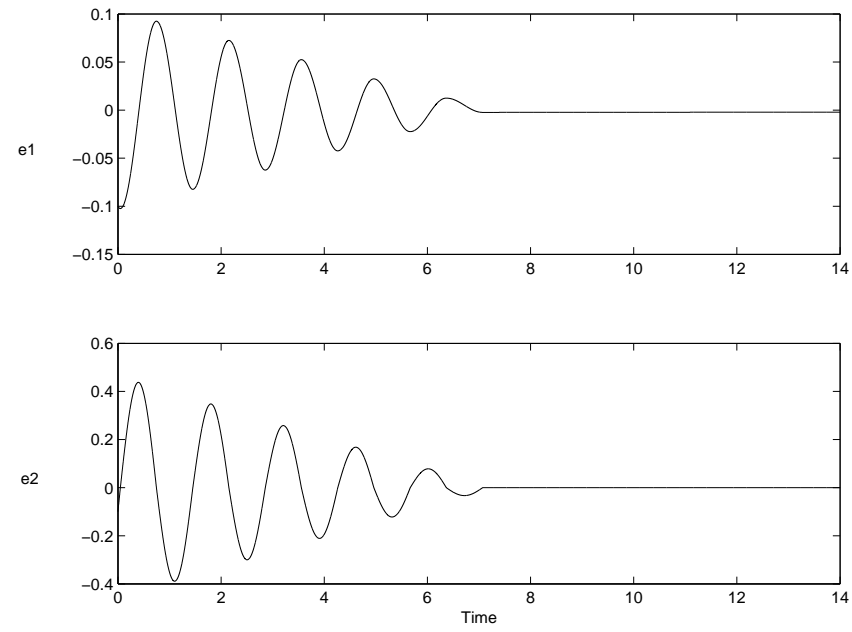

Fig. 2. Simulation of $e_{1}$ and $e_{2}$

\section{ACKNOWLEDGMENT}

The authors gratefully acknowledge discussions with Thierry Floquet on the levitation system.

\section{REFERENCES}

[1] Z. Artstein, "Stabilization with relaxed controls," Nonlinear Anal., vol. 7, no. 11, pp. 1163-1173, 1983.

[2] E. Sontag, "A universal construction of Arststein's theorem on nonlinear stabilization," Systems Control Lett., vol. 13, pp. 117-123, 1989.

[3] E. Mickael, "Continuous selections." Anal. Math., vol. 63, no. 2, pp. 361-382, march 1956.

[4] J. P. Aubin and A. Cellina, Differential Inclusion, S.-V. B. H. N. Y. Tokyo, Ed. Springer-Verlag, Grundlehren der mathematischen Wiissenschaften, 1984, vol. 264.

[5] A. Schinzel, Selected Topics on Polynomials. University of Michigan Press, 1982.

[6] H. K. Khalil, Nonlinear Systems, ser. Third Edition. Upper Saddle River: Prentice-Hall, 2002.

[7] A. F. Filippov, Differential Equations with Discontinuous Righthand Sides, F. M. Arscott, Ed. Dordrecht/Boston/London: Kluwer Academic Publishers, 1988.

[8] D. Shevitz and B. Paden, "Lyapunov stability of nonsmooth system," IEEE Trans. Automat. Control, vol. 39, no. 9, pp. 1910-1914, 1994.

[9] O. Bethoux, T. Floquet, and J. P. Barbot, "Advanced sliding mode stabilization of a levitation system," in European Control Conference, Cambridge, UK, 2003.

[10] G. V. Smirnov, Introduction to the Theory of Differential Inclusions. American Mathematical Society, Graduate Studies in Mathematics, 2002, vol. 41 